\title{
Study regarding the springback of metal sheet parts after bending
}

\author{
Mihail Purcarea*, and Andrei Dumitrescu \\ Politehnica University of Bucharest, Department of Manufacturing Engineering, Bucharest, Romania
}

\begin{abstract}
Plastic deformation of materials is accompanied by elastic deformation. After the bending process, the elastic deformation disappears and the part is changing its dimensions comparing with those of the tool. The change of bent part's dimensions due to the elastic properties of material is called elastic springback. It influences the precision of bent parts, especially the bending angle, and this is why its value should be known in order to take the necessary measures in order to reduce or even annihilate its effects. Its value can be determined with analytical relations, from various nomograms or with experimental relations. Because the analytical relations offer results far from reality, this paper proposes a multivariable relation of springback (dependent variable) as a function of several independent variables obtained on the basis of experimental results, in real working conditions and using the method of regression functions. This method is based on simultaneous variation of considered variables, using a limited number of values in the experimentation domain. A split experimental program was used. The obtained relation is true for any combination of values for considered variables, but, from their variation domains, it can be obtained separately the effects of independent variables on dependent variables.
\end{abstract}

\section{Introduction}

When bending the parts, the plastic deformation is accompanied by an elastic deformation that disappear, after removing the forces which has produced the bending. The part has the dimensions $\alpha_{r}, r_{r}, \varphi_{r}$, different from those of the tool $\left(\alpha_{0}, r_{0}, \varphi_{0}\right)$, as displayed in Figure 1 , where $\alpha, r$ and $\varphi$ are the bending angle, bending radius and supplementary bending angle in the two situations. The modification of the bent part dimensions, because of the material's elastic properties, is called elastic springback or elastic recovery. Springback has influence on the bending angle but also on the bending radius, the biggest influence being on the bending angle.

The value of elastic springback can be determined by analytical relations or is obtained usually with empirical relations or with graphics and nomograms $[1,2,3]$. In all cases, the obtained results are more or less satisfactory, meaning that the results obtained with these relations, graphics or nomograms are different from those obtained by measuring the real processed parts.

\footnotetext{
* Corresponding author: mhlpurcarea@yahoo.com
} 


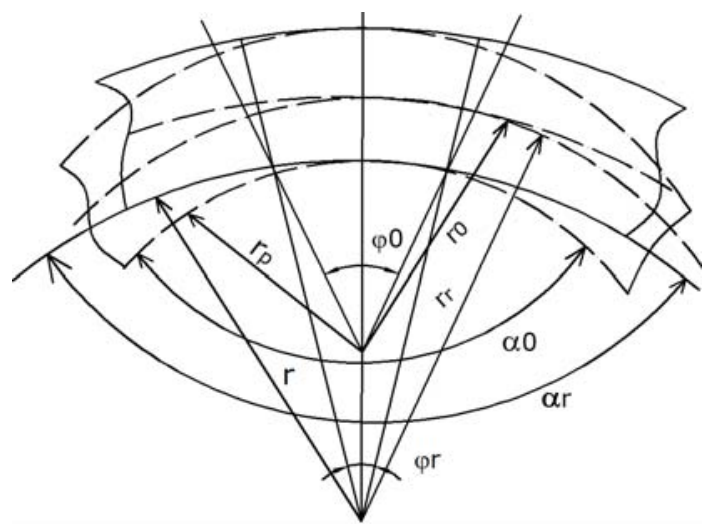

Fig.1. Parameters of bent part before and after removing from the work zone.

These differences arise mainly because of the simplifying hypotheses. For example, in the analytical determination of elastic springback, it is considered that the bending moment characterizes a state of total plastic deformation, when in fact, the state of deformation is an elastic-plastic one with hardening. The importance of analytical-established relations is a theoretical one by establishing parameters that influence the elastic springback, and by establishing the direction effect of their influence on elastic springback.

The elastic springback is defined as the difference between the remnant angle of the part $\alpha_{r}$, after it comes out of the work area, and the angle $\alpha_{0}$ of the tool's active elements.

\section{Relations of elastic springback determined on the basis of experimental results}

In order to eliminate the previously indicated disadvantage, respectively the low precision of analytical relations, the present paper determines some relations obtained from measured values in established working conditions. Experiments carried out in real working conditions should generally be the basis of the validation of process simulation results [4], and therefore of the bending process - the springback phenomenon in this case.

The relations that will be determined will be of multivariable type, respectively dependencies like:

$$
\Delta \alpha=f\left(x_{1}, x_{2}, \ldots, x_{n}\right)
$$

Where:

$\Delta \alpha$ is the dependent variable - elastic springback;

$x_{i}$ - independent variables, with a direct influence upon the elastic springback;

$f$ - the dependency between dependent variable and the independent variables.

Knowledge of quantitative relations between process variables (dependent variables) and their significant variables (independent variables) is very important for studying, researching and optimal conducting of manufacturing processes.

\section{Employed experimental research method}

The experimental research method that was used is the response surface method which considers the relationship between process parameters and characteristic responses of them as surfaces in the dimensional space of variables [5]. In experiments conducted using this 
method, the independent variables are varied simultaneously taking a limited number of values in the considered domain, called levels. This enables the highlighting of existing interactions between independent variables contributing to a more accurate determination of the overall optimum. With this method, the main effects and of higher order and the interactions of independent variables can be determined separately though more independent variables are varied simultaneously. It can be established the order of variables' influence and be excluded those without significant influence. It can be verified the accuracy of the determined mathematical model and the model can be perfected in order to increase the precision of reproduction of experimental data.

Using response surface method in establishing mathematical models of a process generally requires the following steps: choosing the form and the complexity of the proposed mathematical model; programming the experiment; establishing the experimental conditions; conducting the experiment; determining the model's coefficients.

The determined relation (if adequate) displays the following characteristics:

- Can be used (it is consistent) for any combination of values of the independent variables considered in their domain of variation;

- Although the independent variables are varied simultaneously, their effects on the dependent variable can be determined separately when other variables are constant;

- The order of independent variables' influence can be determined and also the variables with no significant influence can be established;

- The precision of the established mathematical model can be determined and the model can be refined in order to increase the precision of reproduction of experimental data;

- The established model can be visualized as a hyper surface in the multidimensional space of independent variables, this being the response surface whose points have as coordinates the corresponding values of process variables.

\section{Variables of the studied process}

The purpose is to establish a relation in the following type:

$$
\Delta \alpha=f(\alpha, g, b, \beta)
$$

Where: $\Delta \alpha$ is the elastic springback and represents the dependent variable [ $\left.{ }^{\circ}\right]$;

$\alpha$ - bending angle $\left[{ }^{\circ}\right]$;

$g$ - thickness of the processed material [mm];

$b$ - width of the bent part [mm];

$\beta$ - angle between the bending line and the direction of part's rolling lines $\left[{ }^{\circ}\right]$.

Variables $\alpha, g, b, \beta$ are the dependent variables of the studied process.

Tables 1 shows the variation levels of independent variables.

Table 1. Independent variables and their levels of variation.

\begin{tabular}{|c|l|c|c|c|c|}
\hline \multicolumn{2}{|c|}{ Independent Variables } & $\begin{array}{c}\boldsymbol{\alpha} \\
{\left[{ }^{\circ}\right]}\end{array}$ & $\begin{array}{c}\boldsymbol{g} \\
{[\mathrm{mm}]}\end{array}$ & $\begin{array}{c}\boldsymbol{b} \\
{[\mathrm{mm}]}\end{array}$ & $\begin{array}{c}\boldsymbol{\beta} \\
{\left[{ }^{\circ}\right]}\end{array}$ \\
\hline \multirow{3}{*}{$\begin{array}{c}\text { Level of } \\
\text { variation }\end{array}$} & $\min (-1)$ & 30 & 0.5 & 10 & 0 \\
\cline { 2 - 6 } & $\operatorname{mean}(0)$ & 90 & 1 & 20 & 45 \\
\cline { 2 - 6 } & $\max (+1)$ & 150 & 1.5 & 30 & 90 \\
\hline
\end{tabular}

The form of dependency type (2) can be, depending on the chosen forms for independent and dependent variables, as follows:

$$
\Delta \alpha=e^{a 0} \cdot \alpha^{a l} \cdot g^{a 2} \cdot b^{a 3} \cdot e^{a 4 \cdot \beta}
$$


Where the variables are polytrophic function type and

$$
\Delta \alpha=a_{0}+a_{1} \alpha+a_{2} g+a_{3} b+a_{4} \beta
$$

Where the variables are of linear multivariable function type, and coefficients $a_{i}, i=0,1,2$, 3,4 are the models' coefficients that will be determined using regression program.

\section{Experimental programs and obtained results}

Fractional experimental programs have been used for four variables $[5,6]$. The tests were performed in the Cold Pressing Lab of Manufacturing Engineering Department, on 6tf Press, using sets of active plates and punches for "V" bending on TDA sheets.

Table 2. Experimental program measured and calculated results, regression analysis.

\begin{tabular}{|c|c|c|c|c|c|c|c|}
\hline $\begin{array}{l}\text { No. } \\
\text { exp. }\end{array}$ & $\begin{array}{l}(\alpha) \\
x 1\end{array}$ & $\begin{array}{l}(g) \\
x 2\end{array}$ & $\begin{array}{l}\text { (b) } \\
x 3\end{array}$ & $\begin{array}{l}\text { ( } \beta) \\
x 4\end{array}$ & $\begin{array}{l}\text { Measured } \\
\text { values }\end{array}$ & $\begin{array}{c}\text { Calculated values } \\
\text { - rel. (3) }\end{array}$ & $\begin{array}{c}\text { Calculated values } \\
\text { - rel. (4) }\end{array}$ \\
\hline 1 & -1 & -1 & -1 & -1 & 2.63 & 2.97 & 3.19 \\
\hline 2 & 1 & -1 & -1 & 1 & 1.47 & 1.64 & 1.58 \\
\hline 3 & -1 & 1 & -1 & 1 & 4.45 & 4.83 & 4.55 \\
\hline 4 & 1 & 1 & -1 & -1 & 1.55 & 1.72 & 1.71 \\
\hline 5 & -1 & -1 & 1 & 1 & 4.37 & 4.86 & 4.53 \\
\hline 6 & 1 & -1 & 1 & -1 & 1.6 & 1.74 & 1.7 \\
\hline 7 & -1 & 1 & 1 & -1 & 4.55 & 5.09 & 4.66 \\
\hline 8 & 1 & 1 & 1 & 1 & 2.5 & 2.82 & 3.06 \\
\hline 9 & 0 & 0 & 0 & 0 & 3.47 & 2.89 & 3.12 \\
\hline 10 & 0 & 0 & 0 & 0 & 3.25 & 2.89 & 3.12 \\
\hline 11 & 0 & 0 & 0 & 0 & 3.42 & 2.89 & 3.12 \\
\hline 12 & 0 & 0 & 0 & 0 & 4.22 & 2.89 & 3.12 \\
\hline \multirow{5}{*}{\multicolumn{5}{|c|}{ Regression coefficients }} & $a_{0}$ & 2.395 & 3.006 \\
\hline & & & & & $a_{1}$ & -0.503 & -0.019 \\
\hline & & & & & $a_{2}$ & 0.242 & 0.745 \\
\hline & & & & & $a_{3}$ & 0.249 & 0.036 \\
\hline & & & & & $a_{4}$ & 0.002 & 0.007 \\
\hline \multirow{6}{*}{\multicolumn{5}{|c|}{ Indicators of regression analysis }} & $R^{*}$ & 0.557 & 0.236 \\
\hline & & & & & $R_{0}$ & 55.3 & 68.22 \\
\hline & & & & & $R_{1}$ & 5.35 & 5.74 \\
\hline & & & & & $\boldsymbol{R}_{2}$ & 0.58 & 0.65 \\
\hline & & & & & $R_{3}$ & 0.61 & 0.62 \\
\hline & & & & & $\boldsymbol{R}_{4}$ & 0.39 & 0.44 \\
\hline
\end{tabular}

The elastic springback was calculated as the difference between the actual angle of active plate and the part's angle after processing. The part's angle was measured with a dial goniometer possessing a precision of 5'. The final value was the mean of three readings.

The experimental program structure, the elastic springback value for each experience, and the regression analysis coefficients and indicators are presented in Table 2. 


\section{Interpretation of results}

It can be observed that:

- both models are adequate, $R^{*}$ coefficients being smaller than $1\left(R^{*}<1\right)$;

- relation (4), by its calculated values, is more close to the measured values $\left(R^{*}{ }_{4}<R^{*}\right)$;

- $\alpha$ variable (bending angle) is highly significant in both cases $\left(R_{l}>1\right)$, the angle increase leading to the decrease of elastic springback $\left(a_{l}<0\right)$;

- variables $g, b, \beta$ (with the meanings presented above) are not statistically significant $\left(R_{2}\right.$, $\left.R_{3}, R_{4}<1\right)$, but their increase leads to the increase of elastic springback $\left(a_{2}, a_{3}, a_{4}>0\right)$.

As was mentioned before, the determined relations (type 3 or 4) are adequate for any combination of values of the independent variables in their experimentation domain. Also, it can be determined the individual influences of each independent variable or group of independent variables on the dependent variable, keeping other variables constant, characterized by analytical or graphical dependencies, as in the following examples:

Considering the relation type (4):

$$
\Delta \alpha=a_{0}+a_{1} \alpha+a_{2} g+a_{3} b+a_{4} \beta
$$

For values at mean level (see Table 1), it is obtained the following invariable mathematical dependency for independent variables $g, b, \beta$ (variable $\alpha$ possessing the highest significance):

$$
\Delta \alpha=f(\alpha)=4,786-0,019 \cdot \alpha
$$

The corresponding graphical dependency (increasing the bending angle leads to the decrease of elastic springback) is displayed in Figure 2.

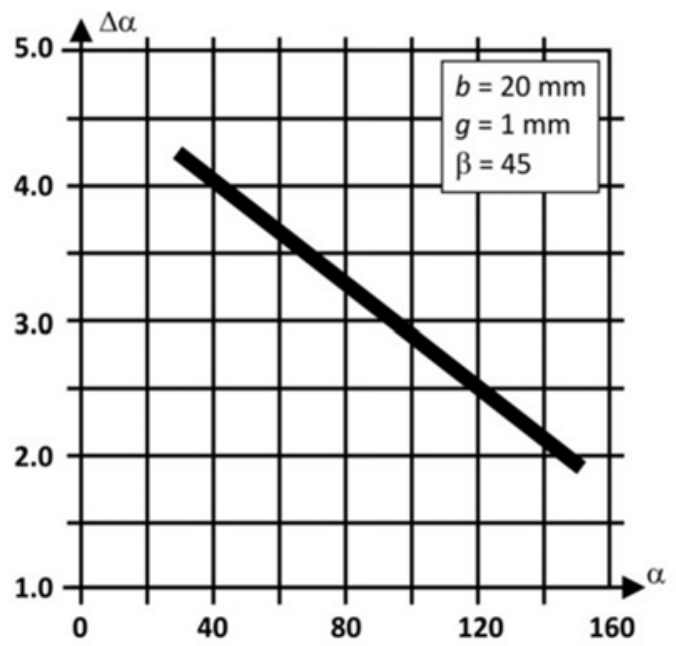

Fig. 2. Variation of springback $\Delta \alpha$ versus bending angle $\alpha$.

For the same relation (4), it can be determined the mathematical and graphical dependencies of two variables, the others having constant values. For example, on the basis of relation (6), in which the independent variables $b, \beta$ have mean values in their variation domain, it was obtained the graphical dependency displayed in Figure 3.

$$
\Delta \alpha=f(\alpha, g)=4,041-0,019 \cdot \alpha+0,745 \cdot g
$$




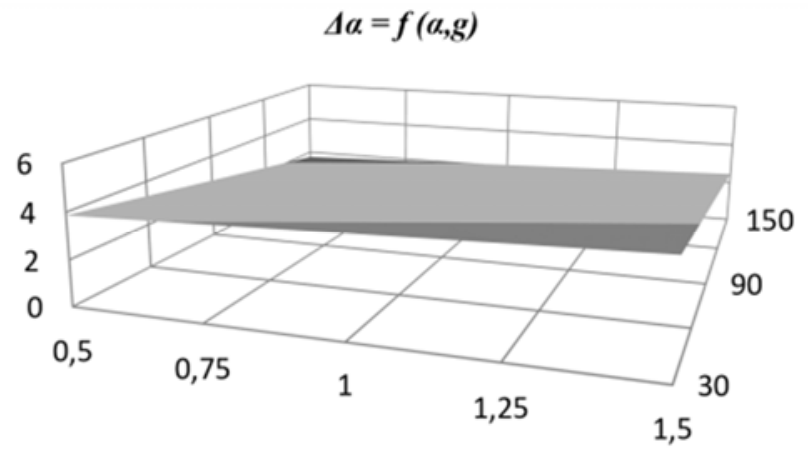

Fig. 3. Variation of springback $\Delta \alpha$ versus bending angle $\alpha$ and thickness of processed material $g$.

Such dependencies (mathematical and graphical) can be obtained for any constant values for the independent variables, provided that these values remain in the domain of variation.

\section{Objectives and Conclusions}

It is envisaged the development of a normative for direct determination of elastic springback in given conditions for the independent variables (in the considered variation domains).

Also, there will be developed annexes with graphical dependencies, with one or two parameters, for elastic springback.

The variation domain for statistically insignificant variables will be enlarged in order to establish more precisely their significance degree. Other influences (material's type, bending radius) on the elastic springback will be studied.

Knowing the precise value of elastic springback is important for part's manufacture with the prescribed bending angle. If elastic springback makes the bending angle out-oftolerance, then constructive and technological measures are taken in order to reduce, even eliminate, the effects of elastic springback.

\section{References}

1. C. Ciocardia, et.al., Cold pressing technology (in Romanian), (EDP, Bucharest, 1991)

2. C. Iliescu, (ed.), Cold Pressing Technology, Elsevier, 1990

3. Gh. Sindila, et.al., Technological systems for cold pressing technology (vol.1) (in Romanian), (BREN Publishing House, Bucharest, 2014)

4. Demeri, M., Lou, M., and Saran, M., SAE Technical Paper 2000-01-2657 (2000)

5. Gh. Sindila, et.al., Manufacturing by cold pressing technology (in Romanian), (BREN Publishing House, Bucharest, 2009)

6. R. Mihail, Introduction in strategy of experimentation with applications in chemical technology (in Romanian), (Stiintifica si Enciclopedica Publishing House, 1976) 